\title{
Petroselinum crispum extract attenuates hepatic steatosis in rats fed with fructose enriched diet
}

\author{
Yuneesha Nair $\mathrm{V}^{1,2}$, Balakrishanan $\mathrm{N}^{2}$, Victor Antony Santiago $\mathrm{J}^{2}$ \\ Faculty of Pharmacy, Bendigo Campus, La Trobe University, Bendigo, Australia. jvictorbiochem@gmail.com
}

\begin{abstract}
Non alcoholic fatty liver disease (NAFLD) is the most common chronic liver disease and ongoing research efforts are focused on understanding the underlying pathophysiology of hepatic steatosis with the anticipation that these efforts will identify novel therapeutic targets. This study investigated the Petroselinum crispum extract in hepatic steatosis in rats fed with fructose enriched diet. Rats were divided into the 4 groups: Group 1 rats received standard pellet diet with corn starch for the entire experimental period of 8 weeks. Group 2 rats received standard pellet diet and $2 \mathrm{gm} / \mathrm{kg}$ body weight crude Parsley leaf ethanol extract for the entire experimental period of 8 weeks. Group 3 rats received modified fructose diet. Group 4 rats received modified fructose diet and $2 \mathrm{gm} / \mathrm{kg}$ crude Parsley leaf ethanol extract. Hepatic function and structure was evaluated in these rats. Modified fructose diet produced dyslipidemia, hepatic steatosis and infiltration of inflammatory cells in the liver and higher plasma hepatic markers. Petroselinum crispum extract reversed metabolic changes such as abnormal hepatic function tests and liver pathology. These results suggested that a non-nutritive role for Petroselinum crispum extract attenuated chronic changes in modified fructose diet induced NAFLD (Tab. 2, Fig. 3, Ref. 43). Text in PDF www.elis.sk.

KEY WORDS: Petroselinum crispum, non alcoholic fatty liver disease, non alcoholic steatohepatitis, lipid-hepatic function indicator.
\end{abstract}

\section{Introduction}

Non alcoholic fatty liver disease (NAFLD) is a condition defined by an excessive fat accumulation in the form of triglycerides (steatosis) in the liver ( $>5 \%$ of hepatocytes histologically) in individuals who do not consume a large amount of alcohol (< $20 \mathrm{~g}$ ethanol/day) (1). Non alcoholic steatohepatitis (NASH) is a progressive form of NAFLD that is diagnosed by histopathological features (2). NAFLD refers to a wide spectrum of liver damage, ranging from simple steatosis to steatohepatitis and cirrhosis (3). Steatosis represents the deposition of fat in hepatocytes, whereas steatohepatitis is the combination of steatosis with hepatic inflammation and fibrosis (4). Development of steatosis involves an impaired lipid metabolism in the liver (5). Insulin resistance, hyperinsulinemia, and oxidative stress are important factors in the development of NASH (6).

NAFLD is rapidly becoming a worldwide public health problem. It is the most common liver disease in the United States and, indeed, throughout the world. Current estimates are that 40-90\%

${ }^{1}$ Faculty of Pharmacy, Bendigo Campus, La Trobe University, Bendigo, Australia, and ${ }^{2}$ Faculty of Pharmacy, Asia Metropolitan University, Cheras, Selangor, Malaysia

Address for correspondence: J. Victor Antony Santiago, Dr, PhD, Faculty of Pharmacy, Asia Metropolitan University, Batu 9, Cheras 43200, Selangor, Malaysia.

Phone: +60.39080 .5888 , Fax: +60.39080 .1995$ of worldwide obese population, $27-34 \%$ of US general population, $20-30 \%$ of Europe general population, $20-40 \%$ of Western countries general population, 20-30\% of Middle East general population and $15 \%$ of Far East general population suffers from NAFLD (7).

Diet is an important contributor to NAFLD, mainly because an excessive energy intake leads to obesity, which in turn increases the risk for NAFLD. A diet based on high cholesterol, high saturated fat and high fructose (cafeteria or fast food type) recapitulates features of NASH with progressive fibrosis (8). Among carbohydrates, specifically fructose contributes to NAFLD progression. Fructose intake has been linked to an increased hepatic fat accumulation, inflammation and fibrosis (9). Fructose has also been associated with both an increase in visceral adipose tissue (10) and plasma triglycerides (11).

The goal of managing NAFLD and NASH is to improve steatosis and prevent fibrosis. There is no standard treatment at present, however, treating risk factors such as insulin resistance, diabetes and obesity remains the focus of managing NAFLD. Currently, lifestyle and dietary interventions, medical treatments, alternative therapies, herbal medicine and surgery are being used to treat risk factors associated with NAFLD (12). Polyphenol rich plants and fruits have been used in folk medicine for treating life style related diseases throughout the world (13).

Peteroselinum crispsum (Parsley) is a bright green, biennial herb, which belongs to the family Apiaceae. Native to the central Mediterranean region (Southern Italy, Algeria, Iran and Tuni- 


\section{$547-553$}

sia) and naturalized elsewhere in Europe, Africa and Asia. It is commonly used as a ganish in soups, salads, meats, vegetables and sauces. Phytochemical screening of Petroselinum crispum (parsley) has revealed the presence of several classes of flavonoids. Its main constituents subsume coumarins, furanocoumarins (bergapten, imperatori), ascorbic acid, carotenoids, flavonoids (kaempferol, quercetin, apigenin and luteolin), apiole, various terpenoic compounds, phenyl propanoids, phathalides, and tocopherol (14-16).

Peteroselinum crispsum (Parsley) has shown health improving effects which includes anti-anemic, menorrhagic effects(17), control of high blood pressure and strengthening the bladder (18), anticoagulant and anti-hyperlipidemic (19), anti-hepatotoxic (20,21), anti-oxidant $(14,22)$, laxative effects $(23)$, normalization of platelet hyper activation (24), treatment of hyperuricemia and gout (25), preventing oxidative stress (25) and reno-protective activity (21).

The present study was aimed to characterize the hepatic biochemical and histological responses to Petroselinum crispum (Parsley) extract against modified fructose diet induced model of non alcoholic fatty liver disease in rats.

\section{Materials and methods}

\section{Plant material and extraction}

Petroselinum crispum (Parsley) plant material was collected from local market in Kuala Lumpur, Malaysia. Plant identification and authentication was done by the botanist Dr. Shamsul Khamis, Co-ordinator, Biodiversity Unit, Institute of Bioscience, Universiti Putra Malaysia, Serdang, Selangor Darul Ehsan, Malaysia where a voucher specimen has been deposited under number: SK 2389/14.

After collection, the leaves of Petroselinum crispum (Parsley) were spattered from other plant parts, dust and then washed with running tap water. The leaves of Petroselinum crispum were shade dried at room temperature for 15 days and coarsely powdered. The powdered leaves were macerated using ethanol at room temperature and extracted for 48 hours with occasional shaking. The soluble ethanol extract were filtered and concentrated to dryness under reduced pressure at $60 \pm 1{ }^{\circ} \mathrm{C}$ in rotary evaporator. Solvent elimination of the resulting ethanol extract was weighed. The percentage yield was calculated using this formula: weight of extract/ original weight $\times 100$ ) of crude Petroselinum crispum extract (PCE). The plant extract was stored in the refrigerator and aliquot of the concentrations was prepared immediately before use.

\section{Animal care and diet}

All the experiment complied with the recommendations and guidelines of the University Animal Ethics Committee and the experimental protocol was approved by the University Animal Ethics Committee (AEC), Asia Metropolitan University, Cheras, Selangor, Malaysia (Reg No: AMU/AEC/HS-FOP/2014/08). Male Wistar rats (150-180 gm, 4-6 weeks old) were supplied by the Central Animal House, Asia Metropolitan University, Cheras, Selangor, Malaysia. They were housed individually in polypropylene cages with paddy husk for bedding at a room temperature of 25 $\pm 2{ }^{\circ} \mathrm{C}$ with relative humidity ( $45 \pm 5 \%$ ) under a 12 -h light-dark cycle. Upon arrival, the rats were allowed 1 week to acclimatize to the new conditions. They were allowed free access to standard rat chow and water ad libitum.

At the start of the experiment, control groups were fed standard pellet diet with corn starch and water ad libitum and other experimental NAFLD groups were fed with modified fructose diet (MFD) which comprises $40 \%$ fructose in diet and $20 \%$ fructose in drinking water. All measures were taken to ensure uniform mixing of the additives of the diet before kneading using a little water.

\section{Experimental timeline}

Rats were randomly assigned into the four groups as follow: Control: Rats received standard pellet diet with corn starch for the entire experimental period of 8 weeks. PCE: Rats received standard pellet diet and $2 \mathrm{gm} / \mathrm{kg}$ body weight crude Parsley leaf ethanolic extract (PCE) for entire experimental period of 8 weeks. MFD: Rats received modified fructose diet (MFD) (40\% of fructose in diet and $20 \%$ of fructose in drinking water) for the total experimental period of 8 weeks. MFD+PCE: Rats received modified fructose $\operatorname{diet}(\mathrm{MFD})$ ( $40 \%$ of fructose in diet and $20 \%$ of fructose in drinking water) and $2 \mathrm{gm} / \mathrm{kg}$ body weight crude Parsley leaf ethanolic extract (PCE) for the total experimental period of 8 weeks).

Group 1: Control: Rats received standard pellet diet with corn starch for the entire experimental period of 8 weeks.

Group 2: PCE: Rats received standard pellet diet and $2 \mathrm{gm} /$ $\mathrm{kg}$ body weight ethanolic extract of parsley (PCE) for entire experimental period of 8 weeks.

Group 3: MFD: Rats received modified fructose diet (MFD) ( $40 \%$ of fructose in diet and $20 \%$ of fructose in drinking water) for the entire the experimental period of 8 weeks.

Group 4: MFD+PCE: Rats received modified fructose diet (MFD) (40\% of fructose in diet and $20 \%$ of fructose in drinking water) and $2 \mathrm{gm} / \mathrm{kg}$ body weight ethanolic extract of parsley (PCE) for the entire experimental period of 8 weeks.

\section{Physiological and metabolic variables}

Metabolic measurements (food intake and water intake) were recorded for all the rats. Water consumption was measured daily to determine fructose dosage. The body weight of each rat was recorded throughout the experimental period.

\section{Terminal experiments}

On the day before euthanasia, the rats were deprived of food for overnight and anaesthetized, and then, rats were killed by decapitation. Blood was collected in heparinized tubes, after $60 \mathrm{~min}$ rest in the supine position, and centrifuged at $160 \times \mathrm{g}$ for $10 \mathrm{~min}$. The plasma was separated and used for the biochemical assays. Liver was carefully cleaned of adherent fat, connective tissue, weighed accurately and used for the histological and biochemical analysis.

\section{Assessment of hepatic structure and function}

Histology of liver

Hepatic tissues were quickly removed after euthanasia, fixed in $10 \%$ buffered formalin for $48 \mathrm{~h}$, dehydrated by pass- 
ing successively in different concentrations of ethanol-water, cleaned in xylene, embedded in paraffin, and sectioned (5-6 $\mu \mathrm{m}$ thickness) using a microtome. Sections were stained with hematoxylin and eosin ( $\mathrm{H}$ and $\mathrm{E})$ and Masson's trichrome and then mounted with neutral deparaffinated xylene (DPX) medium using standard protocol for determination of inflammatory cell infiltration, fat vacuoles and fibrosis.

Hepatic function indicators

The activities of serum aspartate transaminase (AST, EC 2.6.1.1), alanine transaminase (ALT, EC 2.6.1.2), and alkaline phosphatase (ALP, EC 3.1.3.1) were assayed spectrophotometrically according to the standard procedures using commercially available diagnostic kits (Qualichem (Sea) Sdn. Bhd., Selangor Darul Ehsan, Malaysia).

Circulatory and tissue lipids

Lipids were extracted from hepatic tissues by the method of Folch et al (26). HDL cholesterol, total cholesterol, triglycerides in serum and cholesterol, triglycerides in hepatic tissues were quantified spectrophotometrically according to the standard enzymatic procedures using commercially available diagnostic kits (Human Gesellschaft Fur Biochemica und Diagnostica mbH, Weisbaden, Germany).

\section{Statistics}

The data are expressed as the mean \pm S.E.M. Comparisons of the determined variables among all the grouped data for biochemical and physiological parameters were analyzed statistically using one-way analysis of variance (ANOVA) followed by Duncan's multiple range test using the SPSS software package, version 17.01 for windows and statistical significance was defined as $\mathrm{p}<0.05$.

\section{Results}

\section{Body weight changes}

Body weight of the control and experimental rats are shown in Table 1. Body weight was higher in MFD fed rats (group 3) as compared to the control rats (group 1). Administration of PCE at the dose of $2 \mathrm{gm} / \mathrm{kg}$ body weight showed reduced body weight in the MFD+PCE (group 4) treated rats as compared to the MFD alone treated rats (group 3). There were no significant differences between the food intake and water intake in MFD (group 3) and MFD+PCE treated rats (group 4) during the experimental period (Tab. 1).

\section{Histology of liver}

Figure 1 represents the hepatic tissue of control and experimental rats stained with hematoxylin and eosin. MFD alone
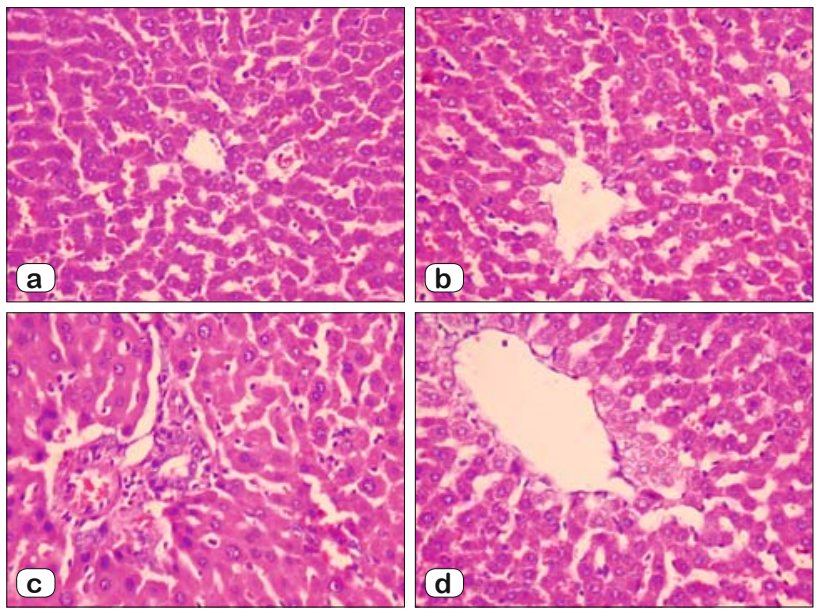

Fig. 1. Photomicrographs of Hematoxylin and eosin stained sections of rat liver from different experimental groups. a) CON: control group, shows normal hepatocytes with central portal vein. b) PCE: Petroselinum crispum extract treated group, shows normal hepatocytes with central portal vein with no pathological alterations. c) MFD: Modified fructose diet fed group, shows microvesicular steatosis, sinusoidal fibrosis and marked inflammatory cell infiltration. d) MFD+PCE; Modified fructose diet and Petroselinum crispum extract treated group, shows reduced microvesicular steatosis, sinusoidal fibrosis and marked inflammatory cell infiltration.
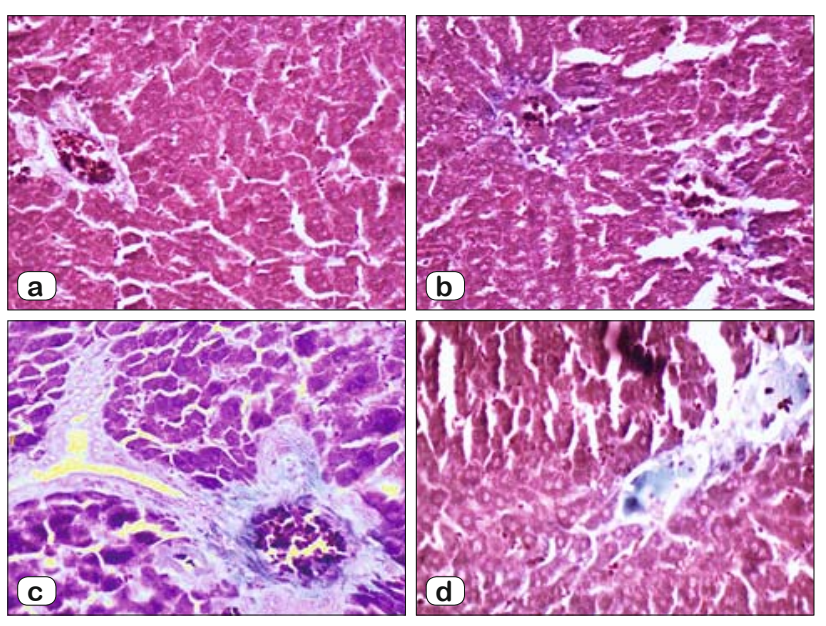

Fig. 2. Photomicrographs of Masson trichrome stained sections of rat liver from different experimental groups. a) CON: control group, b) PCE: Petroselinum crispum extract treated group, c) MFD: Modified fructose diet fed group, d) MFD+PCE; Modified fructose diet and Petroselinum crispum extract treated group, Perisinusoidal, pericellular deposition of collagen and interstitial collagen accumulation appears blue in color.

Tab. 1. General characteristics of control and experimental rats.

\begin{tabular}{|c|c|c|c|c|}
\hline Groups & $\mathrm{CON}$ & PCE & MFD & MFD+PCE \\
\hline Initial body weight (gm) & $185.00 \pm 6.30$ & $190.33 \pm 8.26$ & $186.00 \pm 7.43$ & $188.00 \pm 6.62$ \\
\hline Final body weight (gm) & $224.17 \pm 8.15^{\mathrm{a}}$ & $228.17 \pm 8.15^{\mathrm{a}}$ & $277.00 \pm 10.28^{b}$ & $258.17 \pm 8.15^{\mathrm{c}}$ \\
\hline Liver weight (gm) & $5.45 \pm 0.22^{\mathrm{a}}$ & $5.83 \pm 0.16^{\mathrm{a}}$ & $8.71 \pm 0.34^{b}$ & $7.43 \pm 0.32^{c}$ \\
\hline Hepatic index & $2.43 \pm 0.07^{\mathrm{a}}$ & $2.56 \pm 0.08^{\mathrm{a}}$ & $3.14 \pm 0.08^{b}$ & $2.88 \pm 0.10^{\mathrm{c}}$ \\
\hline Food intake & $15.32 \pm 0.63$ & $15.50 \pm 0.62$ & $16.32 \pm 0.62$ & $15.20 \pm 0.62$ \\
\hline Water intake & $33.21 \pm 1.32$ & $32.21 \pm 1.32$ & $34.31 \pm 1.31$ & $33.25 \pm 1.33$ \\
\hline
\end{tabular}

All the values are expressed as means \pm S.E.M. of 6 rats in each group. Groups not sharing a common superscript letter (a, b and c) differ significantly at $\mathrm{p}<0.05$ (DMRT). 


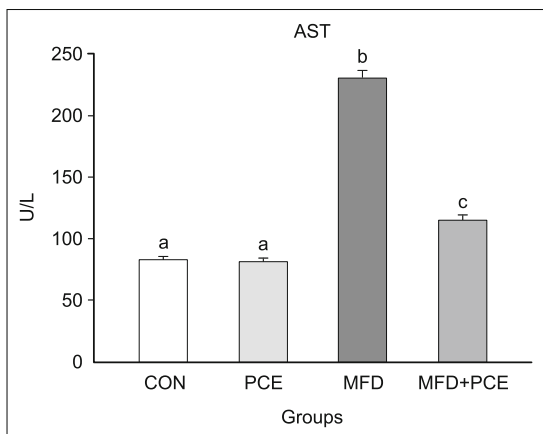

Fig. 3a. Changes in the activities of serum aspartate transaminase (AST) of different experimental groups. a) CON: control group, b) PCE: Petroselinum crispum extract treated group, c) MFD: Modified fructose diet fed group, d) MFD+PCE; Modified fructose diet and Petroselinum crispum extract treated group. Data are expressed as the mean \pm S.E.M. of 6 rats in each group. Groups not sharing a common superscript letter $(a, b$ and $c)$ differ significantly at $p<0.05$ (DMRT).

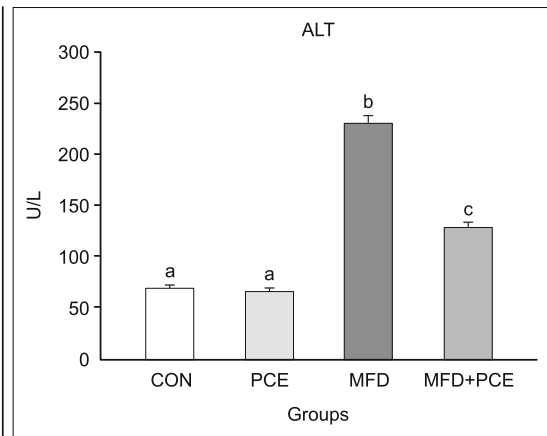

Fig. 3b. Changes in the activities of serum alanine transaminase (ALT) of different experimental groups. a) CON: control group, b) PCE: Petroselinum crispum extract treated group, c) MFD: Modified fructose diet fed group, d) MFD+PCE; Modified fructose diet and Petroselinum crispum extract treated group. Data are expressed as the mean \pm S.E.M. of 6 rats in each group. Groups not sharing a common superscript letter $(a, b$ and $c)$ differ significantly at $p<0.05$ (DMRT).

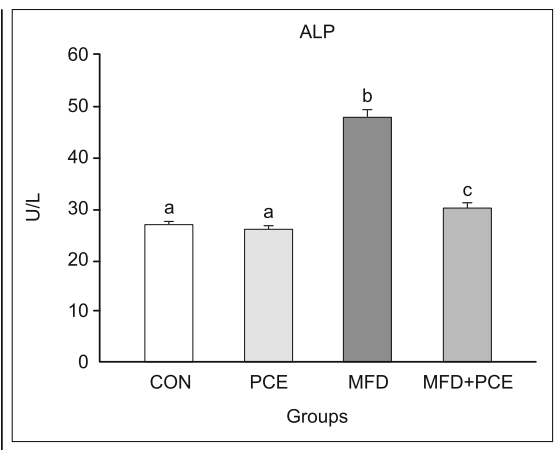

Fig. 3c. Changes in the activities of serum alkaline phosphatase (ALP) of different experimental groups. a) CON: control group, b) PCE: Petroselinum crispum extract treated group, c) MFD: Modified fructose diet fed group, d) MFD+PCE; Modified fructose diet and Petroselinum crispum extract treated group. Data are expressed as the mean \pm S.E.M. of 6 rats in each group. Groups not sharing a common superscript letter (a, $b$ and $c)$ differ significantly at $p<0.05$ (DMRT). treatment (group 3) caused marked changes in the hepatic tissue characterized by microvesicular steatosis, sinusoidal fibrosis and marked inflammatory cell infiltration. Administration of PCE at the dose of $2 \mathrm{gm} / \mathrm{kg}$ body weight revealed reduced microvesicular steatosis, sinusoidal fibrosis and marked inflammatory cell infiltration.

Figure 2 illustrates photomicrographs of Masson's trichrome staining of the hepatic tissue of control and experimental rats. MFD treated rats (group 3) exhibited increased perisinusoidal, pericellular and interstitial collagen accumulation (stained blue). Administration of PCE to MFD fed rats (group 4) revealed reduced collagen accumulation (stained blue) as compared to the MFD alone treated rats (group 3).

\section{Hepatic function indicators}

Hepatic function indicator enzymes such as AST, ALT and ALP activities in the plasma were elevated in MFD fed rats (group 3) as compared to the control rats (group 1). Administration of PCE at the dose of $2 \mathrm{gm} / \mathrm{kg}$ body weight reduced the activities of the hepatic function indicator enzymes in MFD+PCE (group 4) as compared to the MFD (group 3) alone treated rats (Fig. 3).

\section{Circulatory and tissue lipids}

Increased levels (statistically significant at $\mathrm{p}<0.05$ ) of plasma lipids (total cholesterol and triglycerides) with decreased levels of HDL were observed in MFD fed rats (group 3) as compared to the control rats (group 1). Administration of PCE at the dose of 2 $\mathrm{gm} / \mathrm{kg}$ body weight reduced the levels of plasma cholesterol and triglycerides and enhanced the HDL levels in MFD+PCE (group 4) as compared to MFD (group 3) alone treated rats (group 3) (Tab. 2).

Elevated levels of cholesterol and triglycerides in the hepatic tissue were observed in MFD fed rats (group 3) as compared to the control rats (group 1). Administration of PCE at the dose of $2 \mathrm{gm} / \mathrm{kg}$ body weight reduced the cholesterol and triglycerides in MFD+PCE (group 4) as compared to the MFD alone treated rats (group 3) (Tab. 2).

\section{Discussion}

Non alcoholic fatty liver disease (NAFLD) is a major public health issue due to its high prevalence worldwide and potentially serious sequelae. NAFLD is an overarching term for liver histopathology that encompasses a spectrum from simple steatosis to

Tab. 2. Effect of Petroselinum crispum extract on serum lipids and hepatic lipids of control and experimental rats.

\begin{tabular}{|c|c|c|c|c|}
\hline Groups & $\mathrm{CON}$ & PCE & MFD & MFD+PCE \\
\hline \multicolumn{5}{|l|}{ Serum lipids } \\
\hline Triglycerides (mg/dL) & $83.25 \pm 2.59^{\mathrm{a}}$ & $81.74 \pm 2.51^{\mathrm{a}}$ & $229.46 \pm 7.49^{b}$ & $115.27 \pm 4.21^{\mathrm{c}}$ \\
\hline Total cholesterol (mg/dL) & $68.12 \pm 2.41^{\mathrm{a}}$ & $65.92 \pm 2.28^{\mathrm{a}}$ & $154.25 \pm 5.06^{b}$ & $80.24 \pm 2.56^{\mathrm{c}}$ \\
\hline HDL cholesterol (mg/dL) & $55.23 \pm 2.24^{\mathrm{a}}$ & $53.25 \pm 2.14^{\mathrm{a}}$ & $28.22 \pm 1.74^{b}$ & $50.22 \pm 1.91^{\mathrm{a}}$ \\
\hline \multicolumn{5}{|l|}{ Hepatic lipids } \\
\hline Triglycerides (mg/gm tissue) & $3.40 \pm 0.09^{\mathrm{a}}$ & $3.30 \pm 0.09^{\mathrm{a}}$ & $5.86 \pm 0.18^{b}$ & $3.96 \pm 0.12^{\mathrm{c}}$ \\
\hline Cholesterol (mg/gm tissue) & $3.25 \pm 0.09^{\mathrm{a}}$ & $3.12 \pm 0.08^{\mathrm{a}}$ & $6.84 \pm 0.15^{b}$ & $3.99 \pm 0.12^{\mathrm{c}}$ \\
\hline
\end{tabular}

All the values are expressed as means \pm S.E.M. of 6 rats in each group. Groups not sharing a common superscript letter (a, b and c) differ significantly at $\mathrm{p}<0.05$ (DMRT). 
non alcoholic steatohepatitis (NASH). NAFLD is usually caused by two 'hits': the 'first hit' is induced by peripheral insulin resistance, causing hepatic steatosis. The 'second hit' is thought to be caused by reactive oxygen species, inducing vicious cycles of oxidative injury leading to inflammation and fibrosis (27). In this present study, we have shown that Petroselinum crispum (parsley) extract is a possible treatment strategy for modified fructose diet induced rat NAFLD in a rat model. In the present study, we have characterized the changes in liver pathology, lipid composition, and liver function enzymes activities in the rats fed with modified fructose diet. Petroselinum crispum (parsley) extract supplementation significantly reversed the changes induced by feeding modified fructose diet.

The hallmark of NAFLD is triglyceride accumulation in the cytoplasm of hepatocytes. This arises from an imbalance between lipid acquisition (i.e., fatty acid uptake and de novo lipogenesis) and removal (i.e., mitochondrial fatty acid oxidation and export as a component of VLDL particles) (28). Under physiological conditions, the low steady-state triglyceride concentrations in the liver are attributable to a precise balance between acquisition by uptake of non-esterified fatty acids from the plasma and by de novo lipogenesis, versus triglyceride disposal by fatty acid oxidation and by the secretion of triglyceride-rich lipoproteins (28). In the setting of excess carbohydrates, fatty acids are also synthesized de novo within the liver (29). These fatty acids converted into other lipid species, such as glycerolipids, glycerophospholipids and sterols, which can be packaged into very low density lipoprotein (VLDL) particles and secreted from the liver into the plasma (28).

An imbalance between the uptake, synthesis, oxidation, and export of lipids results in excessive fat accumulation in the liver. Elevated serum total cholesterol, triglycerides, and free fatty acids and reduced serum HDL, the indicators of hyperlipidemia, are independent predictors of NAFLD. Increased retention of lipids in the hepatocytes, mostly in the form of total cholesterol, triglycerides and free fatty acid, is known to be the common early trait of NAFLD (30). Moreover, there is a strong link between insulin resistance and excessive deposition of lipids in hepatocytes, which is the hallmark for diagnosis of NAFLD (6). In our present study, accumulation of cholesterol, triglyceride in the hepatocytes was observed in the modified fructose diet fed rats resulting in insulin resistance which is in accordance with the previous findings (28, $31,32)$ and also represents the "first hit" in the pathogenesis of NAFLD (33). Petroselinum crispum (parsley) extract treatment to modified fructose diet treated rats decreased the hepatic fat deposition, especially triglycerides and cholesterol. This could be due to the decreased activities of the key enzymes involved in the synthesis of fatty acid and triglycerides.

Serum aspartate transaminase (AST), alanine aminotransferase (ALT) and alkaline phosphatase (ALP) enzymes activities are well recognized clinical markers of liver damage. Alkaline phosphatase (ALP) is a liver enzyme and its elevation raises the suspicion of infiltrative liver disease. It is elevated in steatohepatitis and advanced NAFLD $(34,35)$. Elevation of AST, ALT, and ALP activities in the plasma is the result of leakage from dam- aged cells and therefore reflects hepatocyte damage (36). Liver transaminases should not be considered as mere biomarkers of NAFLD based solely on blood work. Although elevated liver transaminases can be used as a first step (37). Elevated AST and ALT levels in the absence of other liver diseases may support $\operatorname{NAFLD}(38,39)$, and have been found in up to approximately 50 $\%$ of simple steatosis patients and $80 \%$ of NASH patients (37). However, it is important to note that patients with normal transaminases and liver steatosis on imaging may also have NASH (40). Mild to moderate elevation in the plasma activities of AST and ALT or both is the most common and often the only laboratory abnormality found in patients with NAFLD (41). The AST to ALT ratio appears to be a useful index for distinguishing non alcoholic steatohepatitis from alcoholic liver disease. Although values $<1$ suggest NASH, a ratio of $\geq 2$ is strongly suggestive of alcoholic liver disease, but this ratio increases as fibrosis advances, leading to a loss of its diagnostic accuracy in patients with cirrhotic NAFLD (42). Consistent with the previous findings by Panchal et al (31) and Kawasaki et al (32), our present study of modified fructose diet in rats showed elevated hepatic enzyme (ALT, AST, and ALP) activities, which strongly correlated to NAFLD associated with NASH. Petroselinum crispum (parsley) extract treated rats probably had lower injury and damage to hepatocytes caused by chronic modified fructose diet, as shown by lower serum AST, ALT and ALP activities in plasma.

Diagnosis for NASH is confirmed when a liver pathology shows the presence of perilobular inflammation, or the presence of hepatocyte ballooning, mallory hyaline and acidophil bodies with or without fibrosis (3). Histopathology of modified fructose diet fed rats liver showed features of NAFLD and/ or NASH such as microvesicular steatosis, sinusoidal fibrosis, polymorphonuclear infiltrates, centrilobular inflammation, perisinusoidal, and pericellular deposition of collagen (fibrosis). These evidences demonstrate a systemic pathological change on long term treatment with modified fructose diet, which are features of NAFLD associated with NASH. Petroselinum crispum (parsley) extract treatment reversed the modified fructose diet induced changes.

Efficacy of hepatoprotective drug is essentially dependent on its capability to either reduce the harmful effects or to maintain the normal hepatic physiological mechanisms. The results of the present study revealed that the ethanolic extract of Parsley possesses significant hepatoprotective potential against modified fructose diet induced NAFLD in the experimental model. Hepatoprotective potential of parsley may be attributed to its higher content of flavonoids and vitamin $\mathrm{C}$, which scavenge free radical and antioxidant property, which helps to attenuate hepatic inflammation and fibrosis.

Taken together, the data presented so far indicated that Petroselinum crispum (parsley) extract played a significant role in protecting the hepatic tissue with the most likely mechanisms being decreases in lipid deposition, and improved pathology. The results suggesed that it attenuated hepatic steatosis and improved hepatic pathology, which may serve as a counter regulatory mechanism, to protect the hepatic tissues. Moreover, previous findings with 
Petroselinum crispum (parsley) extract showed beneficial effects against high blood pressure (18), anti-coagulant and anti-hyperlipidemic (19), anti-hepatotoxic $(20,21)$, anti-oxidant $(14,22)$, laxative effects (23), and prevented oxidative stress (25). Also Petroselinum crispum (parsley) extract played a crucial role in lipid lowering effect in hypercholesterolemic rats and protective effects in hepatic tissue with atrophy and vaculation of hepatocyte (43), which fall in line with our present data. These data are clinically very relevant, and ascribe that consumption of Petroselinum crispum (parsley) could prevent early hepatic damage in people prone to NAFLD.

\section{References}

1. McCullough AJ. The clinical features, diagnosis and natural history of nonalcoholic fatty liver disease. Clin Liver Dis 2004; 8: 521-533.

2. Brunt EM, Janney CG, Di Bisceglie AM, Neuschwander-Tetri BA, Bacon BR. Nonalcoholic steatohepatitis: a proposal for grading and staging the histological lesions. Am J Gastroenterol 1999; 94: 2467-2474.

3. Lewis JR, Mohanty SR. Nonalcoholic fatty liver disease: a review and update. Dig Dis Sci 2010; 55: 560-578.

4. Angulo P, Lindor KD. Non-alcoholic fatty liver disease. J Gastroenterol Hepatol 2002; 17: S186-S190.

5. Fabbrini E, Sullivan S, Klein S. Obesity and nonalcoholic fatty liver disease: biochemical, metabolic, and clinical implications. Hepatology 2010; 51: 679-689.

6. Abdelmalek MF, Diehl AM. Nonalcoholic fatty liver disease as a complication of insulin resistance. Med Clin North Am 2007; 91: 1125-1149.

7. World Gastroenterology Organisation (WGO) Global Guidelines, LaBrecque D, Abbas Z, Anania F, Ferenci P, Khan AG, Goh KL, Hamid SS, Isakov V, Lizarzabal M, Pernaranda MM, Rivera Ramos JF, Sarin S, Štimac D, Thomson ABR, Umar M, LeMair A, Krabshuis J. Meta-analyses, Systematic reviews, Practice guidelines: 27 Abstracts. Non alcoholic Fatty Liver Disease and Non alcoholic Steatohepatitis 2012; 1-29.

8. Basaranoglu M, Basaranoglu G, Sabuncu T, Sentürk H. Fructose as a key player in the development of fatty liver disease. World J Gastroenterol 2013; 19: 1166-1172.

9. Vos MB, Lavine JE. Dietary fructose in nonalcoholic fatty liver disease. Hepatology 2013; 57: 2525-2531.

10. Pollock NK, Bundy V, Kanto W, Davis CL, Bernard PJ, Zhu H, Gutin B, Dong Y. Greater fructose consumption is associated with cardiometabolic risk markers and visceral adiposity in adolescents. J Nutr 2012; 142: 251-257.

11. Lê KA, Faeh D, Stettler R, Ith M, Kreis R, Vermathen P, Boesch C, Ravussin E, Tappy L. A 4-wk high-fructose diet alters lipid metabolism without affecting insulin sensitivity or ectopic lipids in healthy humans. Am J Clin Nutr 2006; 84: 1374-1379.

12. Schwenger KJ, Allard JP. Clinical approaches to non-alcoholic fatty liver disease. World J Gastroenterol 2014; 20: 1712-1723.

13. Scalbert A, Manach C, Morand C, Rémésy C, Jiménez L. Dietary polyphenols and the prevention of diseases. Crit Rev Food Sci Nutr 2005; 45: $287-306$.

14. Ozsoy-Sacan O, Yanardag R, Orak H, Ozgey Y, Yarat A, Tunali T. Effects of parsley (Petroselinum crispum) extract versus glibornuride on the liver of streptozotocin-induced diabetic rats. J Ethnopharmacol 2006; 104: 175-181.

15. Pápay ZE, Kósa A, Boldizsár I, Ruszkai A, Balogh E, Klebovich I, Antal I. Pharmaceutical and formulation aspects of Petroselinum crispum extract. Acta Pharm Hung 2012; 82: 3-14.

16. Mahmood S, Hussain S, Malik F. Critique of medicinal conspicuousness of Parsley (Petroselinum crispum): a culinary herb of Mediterranean region. Pak J Pharm Sci 2014; 27: 193-202.

17. Baytop T. Therapy with Medicinal Plants, Past and Present. Istanbul University Publications No. 3255, Istanbul, Istanbul University Press 1984.

18. Simmon JE, Overly ME. A comparative evaluation of Petroselinium cultivars. The spice and Medicinal Plant Digest 1986; 4: 3-47.

19. Yazicioğlu A, Tuzlaci E. Folk medicinal plants of Trabzon (Turkey). Fitoterapia 1996; 67 (4): 307-318.

20. Öztürk Y, Başer KHC, Aydin S. Hepatoprotective (antihepatotoxic) plants in Turkey. Proceedings of the $9^{\text {th }}$ Symposium on Plant Drugs Eskisehir-Turkey 1991; 5: 40-50.

21. Jassim AM. Protective Effect of Petroselinum crispum (parsley) extract on histopathological changes in liver, kidney and pancreas induced by sodium valproate in male rats. Kufa J Vet Med Sci 2013; 4: 20-27.

22. Nielsen SE, Young JF, Daneshvar B, Lauridsen ST, Knuthsen P, Sandström B, Dragsted LO. Effect of parsley (Petroselinum crispum) intake on urinary apigenin excretion, blood antioxidant enzymes and biomarkers for oxidative stress in human subjects. Br J Nutr 1999; 81: 447-455.

23. Kreydiyyeh SI, Usta J, Kaouk I, Al-Sadi R. The mechanism underlying the laxative properties of parsley extract. Phytomedicine 2001; 8: $382-388$.

24. Gadi D, Bnouham M, Aziz M, Ziyyat A, Legssyer A, Legrand C, Lafeve FF, Mekhfi H. Parsley extract inhibits in vitro and ex vivo platelet aggregation and prolongs bleeding time in rats. J Ethnopharmacol 2009; 125: $170-174$.

25. Haidari F, Keshavarz SA, Mohammad Shahi M, Mahboob SA, Rashidi MR. Effects of Parsley (Petroselinum crispum) and its Flavonol Constituents, Kaempferol and Quercetin, on Serum Uric Acid Levels, Biomarkers of Oxidative Stress and Liver Xanthine Oxidoreductase activity in Oxonate-Induced Hyperuricemic Rats. Iran J Pharm Res 2011; 10: 811-819.

26. Folch J, Lees M, Sloane Stanley GH. A simple method for the isolation and purification of total lipids from animal tissues. J Biol Chem 1957; 226: 497-509.

27. Mehta K, Van Thiel DH, Shah N, Mobarhan S. Nonalcoholic fatty liver disease: pathogenesis and the role of antioxidants. Nutr Rev 2002; 60: 289-293.

28. Kawano Y, Cohen DE. Mechanisms of hepatic triglyceride accumulation in non-alcoholic fatty liver disease. J Gastroenterol 2013; 48: 434-441.

29. Cohen JC, Horton JD, Hobbs HH. Human fatty liver disease: old questions and new insights. Science 2011; 332: 1519-1523.

30. Te Sligte K, Bourass I, Sels JP, Driessen A, Stockbrugger RW, Koek GH. Non-alcoholic steatohepatitis: review of a growing medical problem. Eur J Intern Med 2004; 15: 10-21.

31. Panchal SK, Poudyal H, Arumugam TV, Brown L. Rutin attenuates metabolic changes, nonalcoholic steatohepatitis, and cardiovascular remodeling in high-carbohydrate, high-fat diet-fed rats. J Nutr 2011; 141 : 1062-1069. 
32. Kawasaki T, Igarashi K, Koeda T, Sugimoto K, Nakagawa K, Hayashi S, Yamaji R, Inui H, Fukusato T, Yamanouchi T. Rats fed fructose-enriched diets have characteristics of nonalcoholic hepatic steatosis. J Nutr 2009; 139: 2067-2071.

33. Parekh S, Anania FA. Abnormal lipid and glucose metabolism in obesity: implications for nonalcoholic fatty liver disease. Gastroenterology 2007; 132: 2191-2207.

34. Pantsari MW, Harrison SA. Nonalcoholic fatty liver disease presenting with an isolated elevated alkaline phosphatase. J Clin Gastroenterol 2006; 40: 633-635.

35. Verrijken A, Francque S, Mertens I, Talloen M, Peiffer F, Van Gaal $\mathbf{L}$. Visceral adipose tissue and inflammation correlate with elevated liver tests in a cohort of overweight and obese patients. Int J Obes (Lond) 2010; 34: 899-907.

36. Loria P, Lonardo A, Carulli L, Verrone AM, Ricchi M, Lombardini S, Rudilosso A, Ballestri S, Carulli N. Review article: the metabolic syndrome and non-alcoholic fatty liver disease. Aliment Pharmacol Ther 2005; $22: 31-36$.

37. Yan E, Durazo F, Tong M, Hong K. Nonalcoholic fatty liver disease: pathogenesis, identification, progression, and management. Nutr Rev 2007; 65: 376-384.
38. Adams LA, Feldstein AE. Non-invasive diagnosis of nonalcoholic fatty liver and nonalcoholic steatohepatitis. J Dig Dis 2011; 12: 10-16.

39. Pratt DS, Kaplan MM. Evaluation of abnormal liver-enzyme results in asymptomatic patients. N Engl J Med 2000; 342: 1266-1271.

40. Adams LA, Angulo P, Lindor KD. Nonalcoholic fatty liver disease. CMAJ 2005; 172: 899-905.

41. Festi D, Schiumerini R, Marzi L, Di Biase AR, Mandolesi D, Montrone L, Scaioli E, Bonato G, Marchesini-Reggiani G, Colecchia A. Review article: the diagnosis of non-alcoholic fatty liver disease availability and accuracy of non-invasive methods. Aliment Pharmacol Ther 2013; 37: 392-400.

42. Sorbi D, Boynton J, Lindor KD. The ratio of aspartate aminotransferase to alanine aminotransferase: potential value in differentiating nonalcoholic steatohepatitis from alcoholic liver disease. Am J Gastroenterol 1999; 94: 1018-1022.

43. El-Kherbawy GM, Ibrahem ES, Zaki SA. Effects of Parsley and Coriander leaves on hypercholesterolemic rats. The $6^{\text {th }}$ Arab and $3^{\text {rd }}$ International Annual Scientific Conference 2011; 1227-1240.

Received August 28, 2014. Accepted January 10, 2015 